\title{
Fluidic Heterogeneous Microsystems Assembly and Packaging
}

\author{
Wei Zheng, Member, IEEE, Jaehoon Chung, Member, IEEE, and Heiko O. Jacobs, Member, IEEE
}

\begin{abstract}
The nonrobotic fabrication of packaged microsystems that contain nonidentical parts has been accomplished by a directed self-assembly process. The self-assembly process uses geometrical shape recognition to identify different components and subsequent bond formation between liquid solder and metal-coated areas to form mechanical and electrical connections. We applied this concept of shape recognition and subsequent formation of contacts to assemble and package microsystems that contained nonidentical subunits. The self-assembly of three-component assemblies is demonstrated by sequentially adding device segments to the assembly solution including 200- $\mu \mathrm{m}$-sized light-emitting diodes. Six hundred AIGaInP-GaAs light-emitting diode segments self-assembled onto device carriers in 2 min without defects. Encapsulation units self-assembled onto the LED-carrier assemblies to form a three-dimensional (3-D) circuit path to operate the final device. The reported procedure provides a new route to the creation of autonomous heterogeneous microsystems including the realization of autonomous wireless sensor system that requires nonidentical units: CMOS circuitry, Non-CMOS sensor unit for sensing, III-V components for communication, and encapsulation units to form 3-D electrical interconnects. The creation of such systems is being discussed and a proof of concept experiment is being demonstrated.

[1563]
\end{abstract}

Index Terms-Heterogeneous integration, sensor systems, sequential self-assembly, solder- and shape-directed self-assembly.

\section{INTRODUCTION}

$\mathbf{T}$ RADITIONAL manufacturing technologies that focused on assembly include serial pick-and-place, serial wire-bonding, serial packaging, and parallel wafer-to-wafer transfer [1]. As components become smaller, following the trend in miniaturization, conventional robotic methods and assembly lines fail because of the difficulty in building machines that can economically manipulate components in three dimensions that are only micrometers in size. Methods of directed self-assembly have the potential to overcome the limitations of robotic assembly and enable multicomponent microsystems manufacturing in three-dimensions [2]-[7].

Several directed self-assembly methods have been demonstrated to generate functional electrical microsystems. For example, Smith et al. reported shape-directed fluidic methods to position LEDs onto silicon substrates [8]. The authors employed wet-etching properties of silicon and gallium arsenide to form trapezoidal holes and components. The trapezoidal dies prevent upside down assembly and settled in the openings due to gravity

Manuscript received March 29, 2005; revised November 4, 2005. This work was supported by the NSF ECS0300263. Subject Editor D.-I. Cho.

The authors are with the Department of Electrical and Computer Engineering, University of Minnesota, Minneapolis, MN 55455 USA (e-mail: wzheng @ece. umn.edu; hjacobs@umn.edu).

Digital Object Identifier 10.1109/JMEMS.2006.878885 and shape recognition. The process works well on planar substrates and accomplishes filling factors that exceed 99\%. Potential disadvantages are that electrical interconnects require further processing and that the trapezoidal dies are not commonly used in the industry. Srinivasan et al. developed a different process to assemble silicon components and micromirrors onto a gold coated silicon substrate [4], [9]. The authors used hydrophobic patterns on hydrophilic substrates to direct the assembly process of the components; hydrophobic parts attached to the hydrophobic regions to minimize the interfacial free energy. Alignment and bonding were achieved using an adhesive lubrication layer and the defect free assembly of 98 parts, $500 \mu \mathrm{m}$ in size, was accomplished with $0.3^{\circ}$ rotational precision. Gracias et al. demonstrated the formation of 3D electrical networks [10] by adapting a liquid solder directed self-assembly process [11]. The authors demonstrated the defect free assembly of 3-D electrical networks that contained 12 identical polyhedra. The $\sim 1 \mathrm{~cm}$ sized polyhedral carried solder coated sheets of printed circuit board material that was taped onto the faces by hand. Jacobs et al. developed a solder directed self-assembly process to assemble semiconductor dies onto substrates [7]. The process did not use solder on the components that were assembled. Instead, it used metal coated contacts (binding sides) on the dies and solder-based receptors on a substrate to direct the assembly and to form electrical interconnects. Standard die geometries and substrates can be used. The authors demonstrate the assembly of 1500 silicon dies, $300 \mu \mathrm{m}$ in size, and a defect free cylindrical display segment that contained an array of 113 LEDs.

All of the mentioned methods share the advantage of being massively parallel when compared to robotic assembly. Surface tension directed methods [4], [5], [7], [9]-[11] work in 3-D and on curved substrates and can potentially be scaled down to much smaller dimensions; process limitations, parameters, and design rules, however, have not yet been established. The surveyed methods, when compared to robotic assembly lines, lag the ability to realize heterogeneous systems that contain many different parts [12]. While the procedures [4], [5], [7]-[9] allow the positioning of a large number of identical components onto planar and nonplanar surfaces in a massively parallel manner, they are not suitable to realize microelectronic systems that consists of more than one repeating unit. Such heterogeneous systems are difficult to achieve due to the insufficient power of recognition of the existing self-assembly procedures. For example, in shape-directed fluid self-assembly [8], small device components settle into the holes designed to match the shape of larger components; similarly, in surface tension driven self-assembly [4], [5], [7], [9], the binding sites designed for one component will almost always find a partial overlap with a receptor 


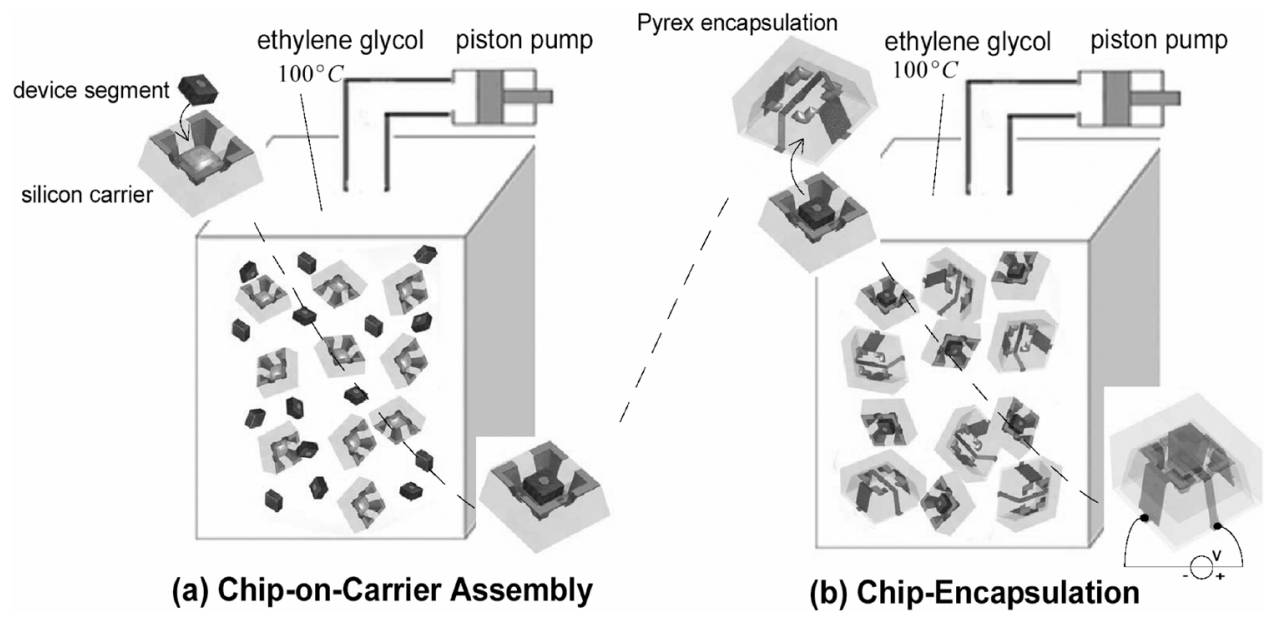

Fig. 1. Fabrication strategy to assemble and package microsystems by shape- and solder-directed self-assembly. Chip-on-carrier assembly (a) and chip-encapsulation (b) are performed in an ethylene glycol solution at a temperature of $100^{\circ} \mathrm{C}$ where the solder is liquid. The illustrated device segment has two contacts: a small circular anode on the front and a large square cathode covering the back. The silicon carrier has a solder-coated area in a tapered opening to host a single semiconductor device segment. The encapsulation unit has five solder-coated copper areas inside a tapered opening to connect to corresponding contact pads on the device and carriers. The agitated components self-assemble in a two-step sequence and form a 3-D circuit path between device layers. (a) Chip-on-carrier assembly and (b) chip-encapsulation

for a different one. As a result the assembly of electrically functional heterogeneous systems that are built using nonidentical components has not been possible. One approach that has been explored in two independent studies is to activate selected receptors to enable batch transfer of desired components onto desired locations [13], [14].

This paper extends research on a directed self-assembly method that has been explored previously to package semiconductor dies [12], [15] and presents recent results on the realization of heterogeneous microsystems that are detached from a common substrate. The first demonstration centers on the packaging of semiconductor dies. The assembled systems contain three nonidentical components: a semiconductor device segment, a carrier, and an encapsulation unit. The second demonstration centers on the assembly of autonomous microsystems that can be activated and interrogated remotely. The presented procedure allows the controlled assembly of three dimensional systems, the registration and electrical contact formation, and the establishment of a three-dimensional (3-D) circuit path that enables surface mount device operation. The assembled systems contain $200 \mu \mathrm{m}$ sized optoelectronic devices. The systems can be seen as compact aggregates that assemble in solution. The aggregates form as designed after adding different components to the solution. Our method combines aspects of previous shape-directed fluidic self-assembly [2], [8] and solder-based self-assemblies [3], [7], [10]. It uses geometrical shape recognition to identify different components and subsequent bond formation between liquid solder and metal coated contacts to form mechanical and electrical connections. This combination provides a greater flexibility in the design of self-assembling systems with minimum defects. Another new element is the use of sequential self-assembly [12]. The process uses a well defined sequence to build the system. Our idea is borrowed from chemical and biological systems that build heterodimers, trimers, and higher aggregates by sequentially adding different molecules. The concept is similar in the sense that every component type can be seen as a different molecule. In our case, however, each component provides a different functionality.

\section{MethodS}

The basic experimental strategy of the self-assembly process is illustrated in Fig. 1 and Fig. 2. Fig. 1 illustrates a first application which centers on the packaging of semiconductor dies whereas Fig. 2 proposes a potential extension to a different application - the assembly of autonomous sensor systems. The differences between the two assembly processes are the number of assembly steps to realize the desired system. Concepts of shape recognition, solder directed self-assembly, and sequential self-assembly are used in both implementations to realize the desired system. We will use Fig. 1 to explain the basic concept where the final structure is formed by a two-step sequence of self-assembly and packaging: (i) chip-on-carrier assembly and (ii) chip-encapsulation. The device segments used in this study are unpackaged cubic $\mathrm{AlGaInP/GaAs} \mathrm{light-emitting} \mathrm{diodes}$ (LEDs) with a side length of $200 \mu \mathrm{m}$ (TK508DR, Tyntek, Taiwan). The LED has two contacts: a small circular anode on the front, and a large square cathode covering the back. Each silicon carrier provided a target site-a 200- $\mu$ m-deep tapered opening - that fits one single LED chip at a time. We integrated a binding site - a $200-\mu \mathrm{m}$-wide square shaped solder-coated area-in the center of the opening for the attachment of the LED chips. During the first self-assembly step, the surface of the liquid solder wets and binds to the gold-coated cathode on the back side of the LEDs. The minimization of the free surface area of the liquid solder drives the assembly into a stable, aligned position. The solder also provides the electrical connection to operate the device and the mechanical bond required to hold the assembly together. We used a low-melting alloy (Y-LMA-117, $\mathrm{mp} \sim 47^{\circ} \mathrm{C}$, Small Parts, Miami Lakes, FL) of bismuth, lead, tin, cadmium, and indium as solder. This solder has been used in previous self-assembly experiment [7], 


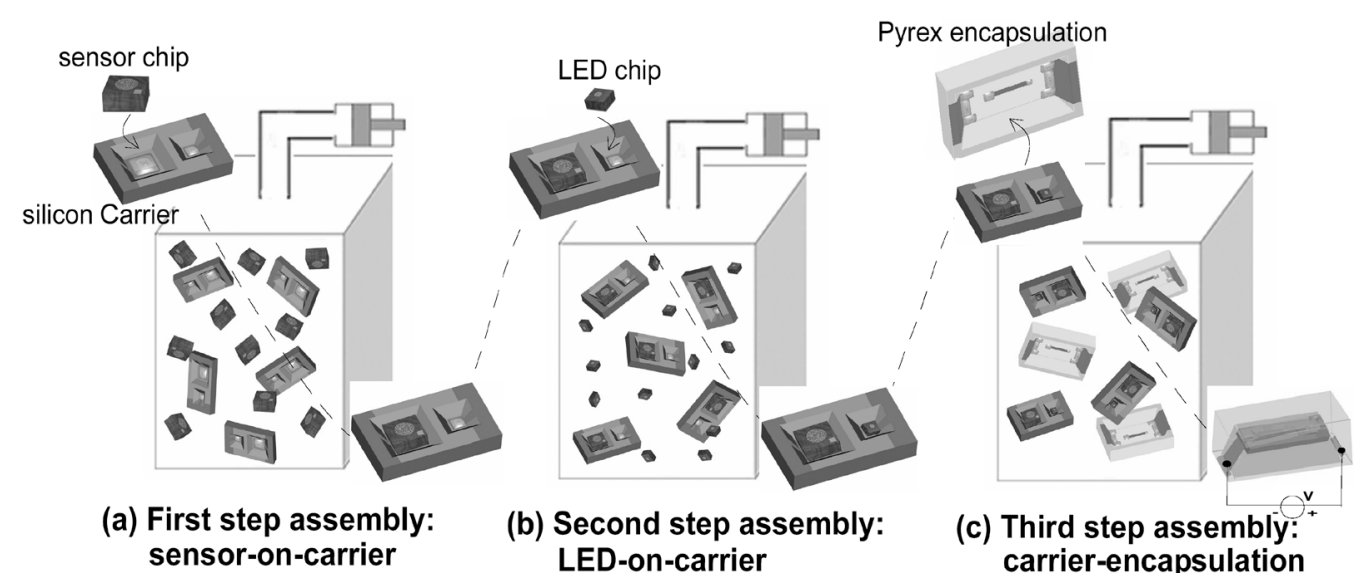

Fig. 2. Fabrication strategy to assemble and package four-component sensor systems containing a silicon carrier, sensor chip for sensing and amplification, light emitting diode for optical communication, and a Pyrex encapsulation unit to package and connect the different components. The assembly is formed by a three-step sequence of self-assembly and packaging: (a) sensor-on-carrier assembly, (b) LED-on-carrier assembly, and (c) carrier-encapsulation. All steps are performed in an ethylene glycol solution with external agitation at a temperature of $100^{\circ} \mathrm{C}$ where the solder is liquid. Each silicon carrier has two differently sized truncated openings with solder-coated areas to capture and assemble a $600 \times 600 \mu \mathrm{m}$ sized sensor chip in the first step and a $200 \times 200 \mu \mathrm{m}$ sized LED chip in the second step, respectively. The encapsulation unit protects the assembled carrier and completes the electrical connections between device layers.

[11] because it has a high surface energy $\left(\sim 400 \mathrm{~mJ} / \mathrm{m}^{2}\right)$ and a sufficiently low melting point.

To protect the assembled LED segments and complete the electrical connections, we fabricated encapsulation units out of $500 \mu \mathrm{m}$-think Pyrex wafers (Corning 7740, Universitywafer, Boston, Massachusetts). Each encapsulation unit carried a $200-\mu$ m-deep tapered opening in the center to recognize the assembled LEDs during the encapsulation process and to distinguish between encapsulation units themselves. Each opening exposed five solder-coated areas to wet and bind to correspondingly-shaped gold-coated areas on the LED and carrier during the second self-assembly step.

Previous solder-based self-assembly systems used a suspension of components in water. To accomplish self assembly, all surfaces must be free from contamination by metal oxides. This is typically accomplished by adding small concentrations of acetic acid ( $\mathrm{pH} 2.5)$ to the assembly solution. This process, however, results in the oxidative dissolution of the solder itself due to two processes: $4 \mathrm{M}+4 \mathrm{nH}^{+}+\mathrm{nO}_{2}=>4 \mathrm{M}^{\mathrm{n}+}+2 \mathrm{nH}_{2} \mathrm{O}$ and $2 \mathrm{M}+2 \mathrm{nH}^{+}=>2 \mathrm{M}^{\mathrm{n}+}+\mathrm{nH}_{2}$. As a consequence, wetting of the copper binding sites with the solder becomes impaired. A number of alternatives to water as the self-assembly solvent were considered. Such a solvent needs to have a high boiling point, and must not react with the solder, copper, glass, silicon, and the photoresist. It must dissolve the acid required to remove surfaces oxides from solder pads and copper contacts, and should not react with this acid. It appears that ethylene glycol and several of its derivatives fulfills these requirements.

The success in directed self-assembly depends in large parts on the agitation that can be provided. Previous research has focused on external agitation by tumbling [3], [10] and shaking the assembly solution [7] to mix components in three dimensions. We tested these forms of agitation and were not successful in mixing the outlined components effectively. The proposed heterogeneous systems contain components out of different materials, dimensions, and densities. Prior agitation methods that used external acceleration did not work in this case which be- comes apparent after consideration of the scaling laws. The following equations are valid for spherical components where $\rho$ and $v$ are the density and velocity difference between the component and a solvent that has a dynamic viscosity $\eta$. For example, the translational force $F_{a}=a x^{3} \rho$ that can be exerted onto the components by external acceleration $a$ scales with the volume $x^{3}$ whereas the drag force $F_{d}=6 \pi \eta v x$ scales linearly with the component size $x$. As a result, it becomes increasingly difficult to agitate components with decreasing size using external agitation since the drag force dominates-components tend to accumulate in the bottom of the assembly container and cannot be assemble effectively.

Instead of trying to overcome the drag force we installed an assembly system that makes use of a pulsating liquid flow and drag to provide agitation. The rational for this approach is as follows: the energy gain due to the surface-tension $\gamma$ scales with the area $x^{2}$ of the involved interfaces and is given as $E_{s}=\gamma x^{2}$ [17]. We find a similar $x^{2}$ relationship for the disordering energy due to a pulsating flow and drag if we consider spherical components with a diameter $x$ and a pulsating laminar flow of velocity $v$; the energy change was calculated by integrating Stokes' law and becomes $E_{d}=3 \pi \eta v x^{2}$. While this is only a first order estimate it illustrates that the ordering energy $E_{s}$ due to the surface tension and the disordering energy $E_{d}$ due to the drag in a liquid flow follow similar scaling laws. We believe that the ability to adjust the disordering energy by increasing the frequency of the pulsating flow together with the similar scaling laws will be particularly useful in the assembly of microsystems that contain components with different dimensions and that have a density that is larger than the density of the assembly solution. Silicon, Pyrex glass, and GaN have a density that is $>2$ times larger than water.

Experimentally the pulsating flow was created using a piston pump (PM6014, Fluid Metering, Inc., Syosset, NY) that expels and retracts liquid through a 2-mm-diameter nozzle that is submerged in the assembly solution. The amount and frequency of liquid that is cycled back and forth can be adjusted between 


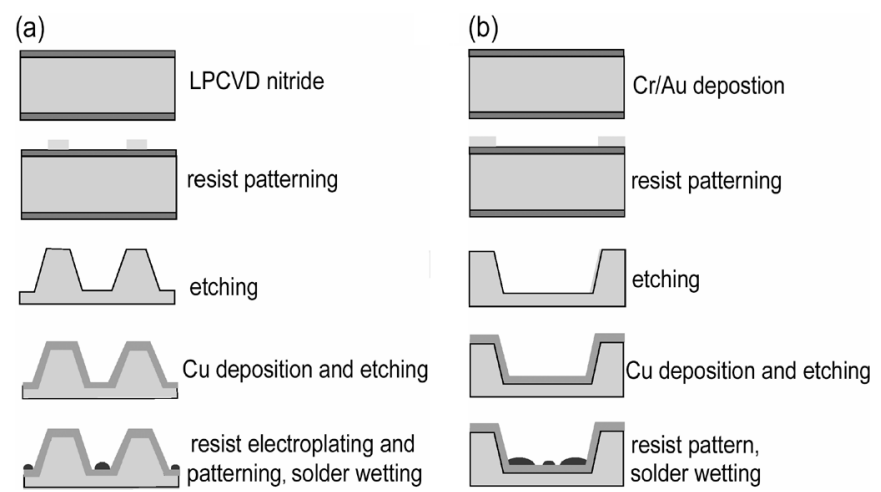

Fig. 3. Micromachining procedure of the self-assembly components. (a) Fabrication of the silicon carriers and (b) fabrication of Pyrex encapsulation units.

1-1.5 mL, and $0-10 \mathrm{~Hz}$, respectively. The assembly was carried out inside a rectangular glass container $(12 \mathrm{~mm}$ on each side and $45 \mathrm{~mm}$ high) filled with $4.5 \mathrm{~mL}$ of ethylene glycol at a temperature of $100^{\circ} \mathrm{C}$. The advantages when compared with tumbling or external acceleration [18] were confirmed experimentally. We observed good mixing of components with different densities while maintaining an adjustable balance between the disordering and ordering energies for components with different dimensions.

\section{FABRICATION}

The procedures to fabricate the silicon carriers, Pyrex encapsulation unit, and patterned solder drops on the three-dimensionally shaped units are described in Fig. 3.

\section{A. Fabrication of the Silicon Carriers}

A p-type $280-\mu \mathrm{m}$-thick Si wafer (Virginia Semiconductor, Fredericksburg, Virginia) was coated with LPCVD nitride to form a $100 \mathrm{~nm}$ thick layer. The substrate was primed with hexamethyldisilazane and spin-coated with photoresist (Microposit 1813, Shipley, Phoenix, Arizona). After a soft-bake at $105^{\circ} \mathrm{C}$ for 1 minute, the substrate was exposed to UV light through a dark field mask. The photoresist was developed in 1 MIF351: $5 \mathrm{H}_{2} \mathrm{O}$ developer for about $15 \mathrm{~s}$. The exposed nitride area was etched in STS etcher for $2 \mathrm{~min}$; the exposed silicon area was etched in $\mathrm{KOH}(45 \%)$ at $80^{\circ} \mathrm{C}$ for $4.5 \mathrm{~h}$ resulting in a 200- $\mu \mathrm{m}$-deep tapered opening. After etching, the silicon wafer was coated with $25 \mathrm{~nm}$ Ti and $800 \mathrm{~nm}$ copper using e-beam evaporator. Shipley Eagle 2100 photoresist was electroplated on the wafer using a dc voltage $(50 \mathrm{~V})$ for $30 \mathrm{~s}$ at $35^{\circ} \mathrm{C}$. After a soft-bake at $80^{\circ} \mathrm{C}$ for $2 \mathrm{~min}$, the substrate was exposed to UV light through the second mask for 60 seconds. The Eagle 2100 photoresist was developed in 1 Eagle 2005 developer: $24 \mathrm{H}_{2} \mathrm{O}$ at $38^{\circ} \mathrm{C}$ for $2 \mathrm{~min}$. The exposed copper was etched in an aqueous ferric chloride solution ( $1.4 \mathrm{~g}$ of $\mathrm{FeCl}_{3}$ per milliliter of $\mathrm{H}_{2} \mathrm{O}$, $\mathrm{pH}$ 1.3) and the Ti was etched in 10:1 buffered oxide etchant. After removing the Eagle 2100 photoresist in acetone, another layer of Eagle 2100 photoresist was electroplated using a dc voltage $(50 \mathrm{~V})$ for $30 \mathrm{~s}$ at $35^{\circ} \mathrm{C}$ to form the solder-coated receptors. The photoresist was exposed and developed using the same conditions as described before. The exposed copper squares were coated with solder by immersing the substrate into a solder bath.
Finally, the wafer was diced using an automated dicing saw to obtain the silicon carriers. The remaining photoresist was removed in acetone to expose the binding sites.

\section{B. Fabrication of Pyrex Encapsulation Units}

A 500- $\mu \mathrm{m}$-thick Corning 7740 wafer (Universitywafer, Boston, Massachusetts) was coated with $25 \mathrm{~nm} \mathrm{Cr}$ and $250 \mathrm{~nm} \mathrm{Au}$ using an e-beam evaporator. The substrate was primed with hexamethyldisilazane, and spin-coated with photoresist (Microposit 1813, Shipley, Phoenix, AZ). After a soft-bake at $105^{\circ} \mathrm{C}$ for $1 \mathrm{~min}$, the substrate was exposed to UV light through a dark field mask. The photoresist was developed in MIF-351 1:5 developer for about $15 \mathrm{~s}$. The metal layers were etched using $4 \mathrm{KI}: 1 \mathrm{I}_{2}$ : $40 \mathrm{H}_{2} \mathrm{O}$ for $\mathrm{Au}$ and $1 \mathrm{HCl}$ : 1 Glycerol : $3 \mathrm{H}_{2} \mathrm{O}$ for $\mathrm{Cr}$. The exposed glass area was etched in $20 \mathrm{HF}(49 \%): 14 \mathrm{NHO}_{3}(69 \%): 66 \mathrm{H}_{2} \mathrm{O}$ for 5 h. After removing the metal layer, the glass wafer was coated with $25 \mathrm{~nm}$ Ti and $800 \mathrm{~nm}$ copper using an e-beam evaporator. The Shipley Eagle 2100 photoresist was electroplated on the wafer using a DC voltage $(50 \mathrm{~V})$ for 30 seconds at $35^{\circ} \mathrm{C}$. After a soft-bake at $80^{\circ} \mathrm{C}$ for $2 \mathrm{~min}$, the substrate was exposed to UV light through the second mask for 60 seconds. The Eagle 2100 photoresist was developed in 1 Eagle 2005 developer: 24 $\mathrm{H}_{2} \mathrm{O}$ at $38^{\circ} \mathrm{C}$ for $2 \mathrm{~min}$. The exposed copper was etched in an aqueous ferric chloride solution $\left(1.4 \mathrm{~g}\right.$ of $\mathrm{FeCl}_{3}$ per milliliter of $\mathrm{H}_{2} \mathrm{O}, \mathrm{pH} 1.3$ ) and the Ti was etched in 10:1 buffered oxide etchant. After removing the Eagle 2100 photoresist in acetone, the Shipley 1805 photoresist was spun on the substrate and patterned to expose the copper area for solder wetting. The opening area was coated with the low-melting point solder by immersing the substrate into a solder bath. Finally, the wafer was diced to obtain the Pyrex encapsulation units. The remaining photoresist was removed in acetone to expose the binding sites.

\section{RESUlTS AND DiscusSIONS}

Fig. 4 illustrates the experimental realization of the two-step: chip-on-carrier assembly and chip-encapsulation process. For the chip-on-carrier assembly [see Fig. 4(a)], we added 3000 LED chips and 600 carrier units into the heated assembly solution and agitated the pieces using the turbulent liquid flow. We obtained a yield of $100 \%$ - that is, all 600 carriers had captured an LED device segment - in $2 \mathrm{~min}$. To prepare for the encapsulation process [see Fig. 4(b)], we removed the excess LEDs by filtration through a $500-\mu \mathrm{m}$ mesh filter and added 200 encapsulation units to the assembly container. The excess LEDs were reused in later experiments. The yield of the encapsulation process was $97 \%$, which means that $3 \%$ of the devices did not function as expected. We reproduced the self-encapsulation process several times and found that variations in the height of up to $\sim 20 \mu \mathrm{m}$, and lateral misfits between the anode of the LEDs and the top electrode of up to $\sim 40 \mu \mathrm{m}$, were tolerated; beyond those limits the devices would not function. Small deviations were compensated by the reflow of solder and the repositioning of the LED, carrier, and encapsulation units. Deviations from the permissible range of sizes and positions resulted from variations in the size of the LEDs $(\sim 30 \mu \mathrm{m})$, and shape, thickness, and location of the solder-coated areas. In some cases we 

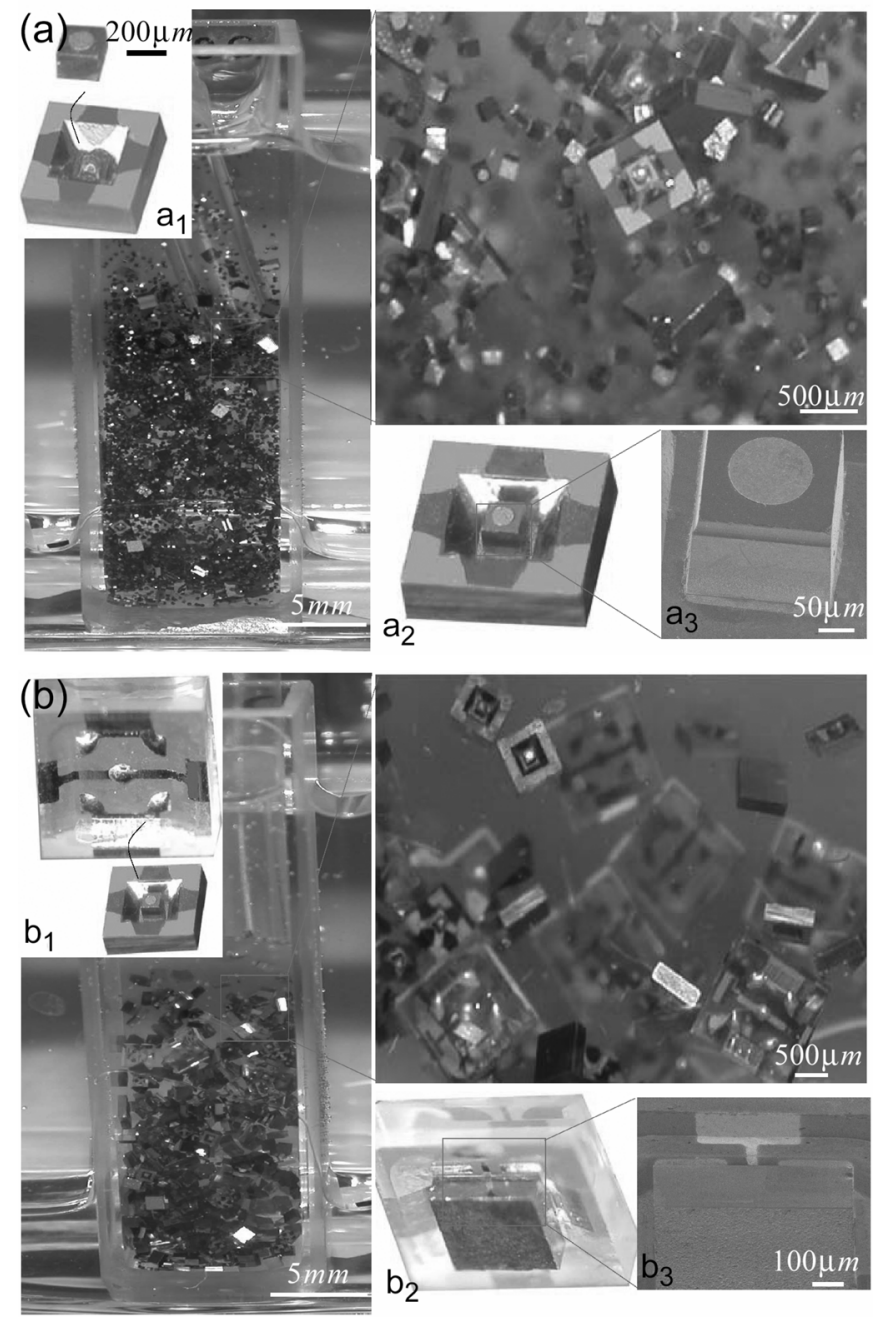

Fig. 4. Experimental realization of LED chips that were assembled and packaged. The insets show device components before $\left(a_{1}, b_{1}\right)$ and after $\left(a_{2}, b_{2}\right)$ each assembly step. The scanning electron micrographs $\left(a_{3}, b_{3}\right)$ illustrate the alignment between components.

also found solder-based areas on the components that were only partially coated with solder, and components with partially detached metal areas as a source of defects. The overall yield of the process is currently $97 \%$ and can be increased by further removing the imperfection in the manufacturing of the components. These defects are not inherent to the self-assembly process itself, but were caused by imperfections in the manufacturing of the components.

Besides the AlGaInP/GaAs LED segments, we successfully assembled and packaged two terminal blue AlGaN/GaN LEDs (LG Innotek, Korea). The contacts on the blue LEDs were located on the same side of the $375 \times 330 \mu \mathrm{m}$ wide and $80 \mu \mathrm{m}$ tall device segments. The dimensions of the final assembly were $800 \mu \mathrm{m} \times 800 \mu \mathrm{m} \times 500 \mu \mathrm{m}$. The procedure and yield ( 97\%) of the assembly and packaging of these devices was similar to that described with the red emitting LED segments. We tested the functionality by hand mounting the devices on a printed circuit board as shown in Fig. 5.

Defects inherent to self-assembly processes are most commonly related to local energy minima in the space of possible conformations or to an insufficient overall energy minimum representing a stable assembly under agitation. An effective self-as-

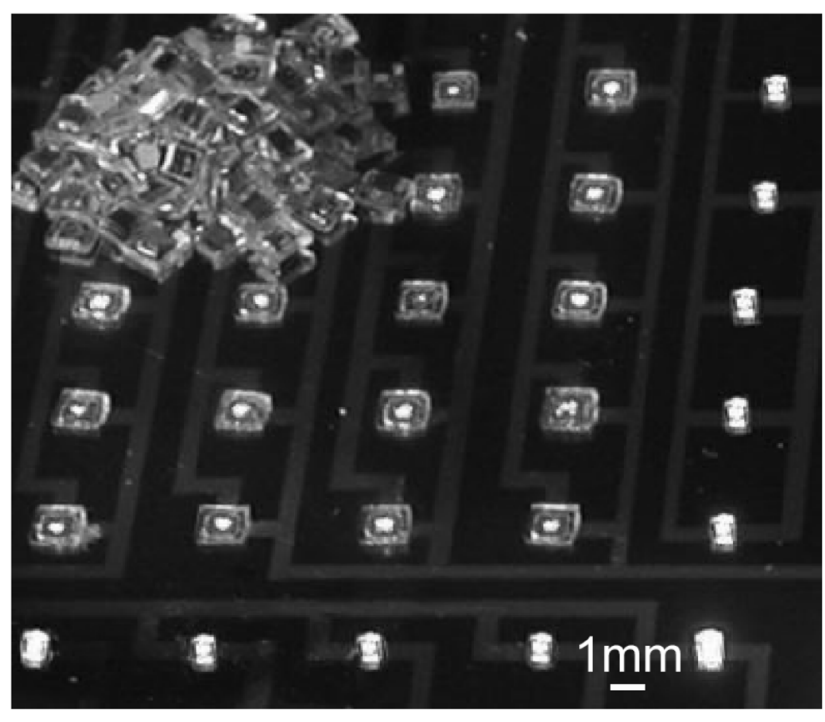

Fig. 5. Testing of LED chips that have been assembled and packaged.

sembly system will exhibit a clear global energy minimum for the desired assembly configuration, while avoiding (as much as possible) local minima or regions of low energy gradient corresponding to undesired configurations and defects [5].

The combination of surface tension directed self-assembly with geometrical confinement can remove defects effectively. Fig. 6 illustrates this idea using a square shape component. The contact pad layout might be given by the manufacturer of the device. Without geometrical confinement four possible assemblies are apparent, three of which are incorrect orientations that occur due to a partial overlap between receptors and binding sites. Introducing the elements of geometrical confinement and shape recognition is effective in removing such defects [see Fig. 6(b)]. Our design approach follows this basic concept and enables the removal of local energy minima and associated defects completely by introducing and optimizing the 2.5-dimensional shapes. The approach does not require exact complementary 3-D shapes. As a design rule sufficient confinement needs to be provided to prevent undesired overlap between the receptor sites and binding sites of the components that are involved. The use of a sequence provides additional flexibility in the design and allows us to maintain simple components that can be manufactured by micromachining. Our design followed this basic procedure. During the course of the work we optimized the overall energy minima by changing the size and shape of the solder-coated areas and the metallic binding sites. Early designs for the encapsulation units could not sustain high levels of agitation; defects were created by component impaction removing previously assembled LEDs and encapsulation units from the carriers. Fast and stable assemblies were accomplished by increasing the binding sites to cover $>30 \%$ of the surface. The symmetry of the design influences the speed of the self-assembly process as well. The presented design has a four-fold symmetry and assembled faster than designs that presented a two-fold symmetry.

We are currently in the process of extending this concept to assemble more complex heterogeneous microsystems. We use the term heterogeneous microsystems to refer to systems that require integration of components that cross traditional 
(a) conformations without shape confinement

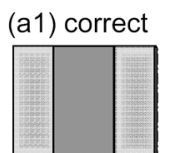

(a2) incorrect local minimum

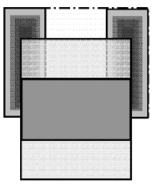

(b) effect of shape confinement

(b1) correct, 100\%

2-fold symmetry

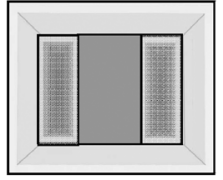

(b2) correct, $100 \%$ 4-fold symmetry

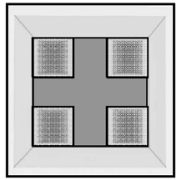

(a3) incorrect, local minimum

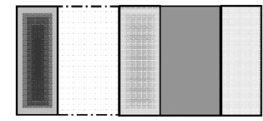

(a4) incorrect, local minimum

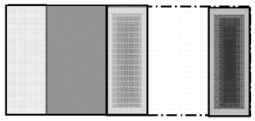

photograph of b1

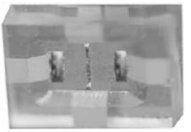

photograph of b2

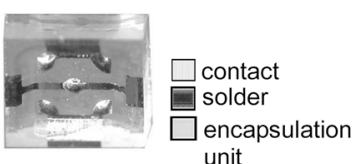

Fig. 6. Effect of shape confinement on assembly conformations and defects. (a) Components with two contact pads assemble on a flat substrate with four stable orientations including three that represent defects. (b) Shape confinement is used to rule out partial overlap between binding sites. The photographs show the experimental realization of packaging elements with a two- and fourfold symmetry. (a) Conformations without shape confinement and (b) effect of shape confinement.

length scales and material boundaries. Heterogeneous systems combine devices that cannot be integrated monolithically. Good examples are miniaturized autonomous sensor systems that contain a number of different parts: CMOS circuitry for processing, Non-CMOS sensor units for sensing, III-V components for communication, and encapsulation units to form 3D electrical interconnects. As a first example in this direction we demonstrate the assembly of a system that contains a silicon carrier element with a simple passive circuit, a sensor chip for sensing and amplification, an III-V light emitting diode for optical communication, and a Pyrex encapsulation unit to package and connect the different components (Fig. 2). The procedure to fabricate the silicon carriers, Pyrex encapsulation unit, circuits, and patterned solder drops on the three-dimensionally shaped units is similar to the fabrication steps described in Fig. 3. The self-assembled system with all the components is illustrated in Fig. 7. Each silicon carrier had two differently sized truncated openings with solder-coated areas: a $200 \times 200 \mu \mathrm{m}$ tapered opening to host a single LED chip, and a $600 \times 600 \mu \mathrm{m}$ tapered opening to host a sensor chip. As sensor chips, we used $\mathrm{P} / \mathrm{N}$ photodiode based detector with an integrated BJT transistor amplifier (ST0124, Three Five Compounds, New York, NY). The $600 \times 600 \mu \mathrm{m}$ sized sensor chips assembled onto the silicon carriers during the first self-assembly step. The sensorchips detect and amplify optical radiation in the visible and near infrared spectral range. A second self-assembly
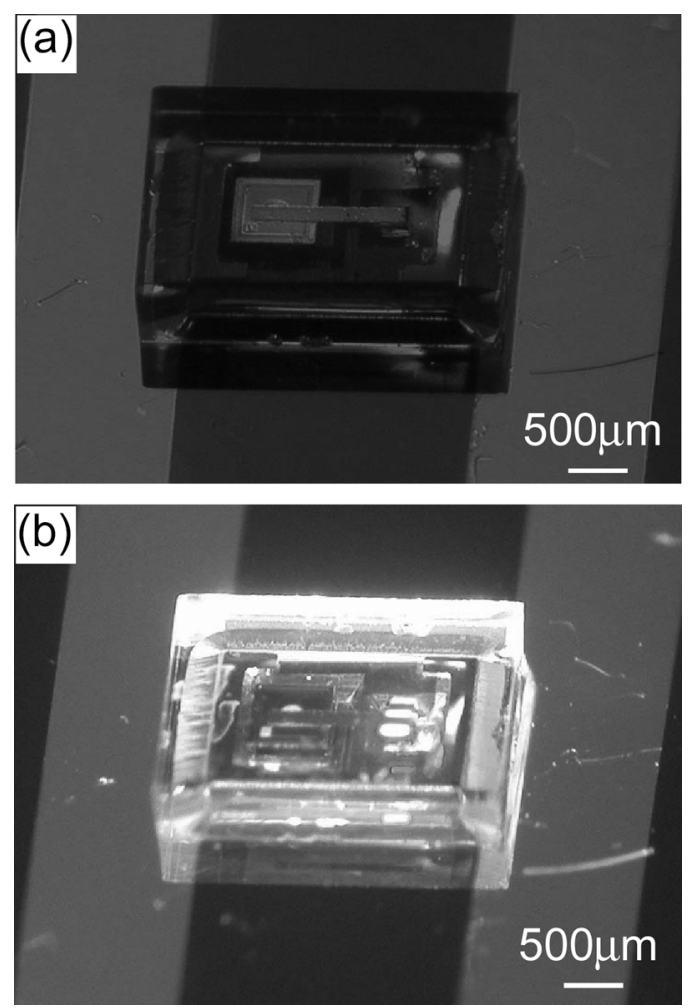

Fig. 7. Testing of a four-component sensor module that can be activated and interrogated remotely and that was assembled and packaged by directed 3-D self-assembly using a three-step self-assembly sequence. (a) Shows the system in the OFF state and (b) shows the ON state where the system is activated and interrogated through remote optical radiation.

step was used to assemble the LEDs onto the silicon carriers. During the third self-assembly step we added encapsulation units to the assembly solution. The encapsulation units protect the assembled components and more importantly complete the electrical connections without the need of a wirebonder. In all steps the minimization of the free surface area of the liquid solder drove the assembly into a stable, aligned position. The functionality of the final structure was tested by hand mounting the assembled sensor modules on a printed circuit board. The self-assembled system is designed to form a transponder: 1) incoming optical radiation is detected by the phototransistor enabling remote activation of the system, 2) the BJT transistor amplifier amplifies the signal to drive the LED, and 3) the LED responds back to the remote interrogator. The results on the autonomous sensor systems should be seen as a proof of concept experiment. It is the first demonstration of this kind and we have not yet started to self-assemble such systems in large quantities. We also do not know what the ultimate complexity could be in terms of dimension, number of components, and number of interconnects that could be created.

\section{CONCLUSION}

We have demonstrated the self-assembly and packaging of microsystems using a directed 3-D self-assembly technique that combines existing concepts of geometrical shape recognition and site specific wetting and binding involving liquid solder with a new concept of sequential self-assembly. The technique is tailored to enable the realization of heterogeneous 3-D microsystems that contain nonidentical parts, and of connecting them 
electrically, where the shape provides the ability to recognize different component, where the solder provides both the driving force, the electrical, and mechanical connections, and where the sequence provides the ability to build complex systems.

We believe the three-fold combination provides, for the first time, a route to the realization of complex systems. The design capability of "single energy minimum" systems is greatly enhanced; many solutions become available to solve a given design problem. The concept could find application in the MEMS community and in the realization of systems that contain sensors, actuators, or microfluidic elements. The ability to assemble multiple components in two- or three dimensions will enable the creation of improved and entirely new sensor systems that go beyond the capacities of current micromachining and microassembly. A variety of systems could be built using the same general approach. Sensor modules and communication elements could be exchanged and higher performance elements could be integrated. The advantage of this process and self-assembly in general over conventional robotic methods is that it is highly parallel. As a result a reduction in the assembly costs can be expected when large quantities are needed. It might also be useful in applications where only small quantities of systems need to be realized and where the large capital investment of acquiring and maintaining a robotic assembly line cannot be justified. With the elimination of robotic assembly lines, however, comes an increased effort in the manufacturing of the parts that can effectively direct the self-assembly. This additional effort can be limited if conventional surface micromachining is used to realize the components, which was the case in our example. The use of nonconventional lithographic methods where the components can be patterned on all faces, however, would provide even greater flexibility in the creation of 3-D systems that cannot be fabricated otherwise. The concept could also be combined with programmable self-assembly concept that activate receptors sequentially [14].

\section{REFERENCES}

[1] M. B. Cohn, K. F. Bohringer, J. M. Noworolski, A. Singh, C. G. Keller, K. Y. Goldberg, and R. T. Howe, "Microassembly technologies for MEMS," Proc. SPIE, vol. 3512, pp. 2-16, 1998.

[2] H. J. J. Yeh and J. S. Smith, "Fluidic self-assembly for the integration of gas light-emitting diodes on si substrates," IEEE Photon. Technol. Lett., vol. 6, pp. 706-708, 1994.

[3] M. Boncheva, D. H. Gracias, H. O. Jacobs, and G. M. Whitesides, "Biomimetic self-assembly of a functional asymmetrical electronic device," Proc. Nat. Acad. Sci. USA, vol. 99, pp. 4937-4940, 2002.

[4] U. Srinivasan, M. A. Helmbrecht, C. Rembe, R. S. Muller, and R. T. Howe, "Fluidic self-assembly of micromirrors onto microactuators using capillary forces," IEEE J. Sel. Topics Quantum Electron., vol. 8, pp. 4-11, 2002.

[5] K. F. Böhringer, U. Srinivasan, and R. T. Howe, "Modeling of fluidic forces and binding sites for fluidic self-assembly," presented at the IEEE International Conference on Micro Electro Mechanical Systems (MEMS), Interlaken, Switzerland, 2001, unpublished.

[6] J. Fang, K. Wang, and K. F. Böhringer, "Self-assembly of micro pumps with high uniformity in performance," presented at the Solid State Sensor, Actuator, and Microsystems Workshop, Hilton Head Island, SC, 2004, unpublished.

[7] H. O. Jacobs, A. R. Tao, A. Schwartz, D. H. Gracias, and G. M. Whitesides, "Fabrication of a cylindrical display by patterned assembly," Science, vol. 296, pp. 323-325, 2002.

[8] J. S. Smith and H. J. J. Yeh, "Method and Apparatus for Fabricating Self-Assembling Microstructures," U.S. Pat. 5 824 186, 1998.

[9] U. Srinivasan, D. Liepmann, and R. T. Howe, "Microstructure to substrate self-assembly using capillary forces," J. Microelectromech. Syst., vol. 10 , pp. 17-24, 2001.
[10] D. H. Gracias, J. Tien, T. L. Breen, C. Hsu, and E. M. Whitesides, "Forming electrical networks in three dimensions by self-assembly," Science, vol. 289, pp. 1170-1172, 2000.

[11] T. L. Breen, J. Tien, S. R. J. Oliver, T. Hadzic, and G. M. Whitesides, "Design and self-assembly of open, regular, 3D mesostructures," Science, vol. 284, pp. 948-951, 1999.

[12] W. Zheng, P. Buhlmann, and H. O. Jacobs, "Sequential shape-andsolder-directed self-assembly of functional microsystems," Proc. Nat. Acad. Sci. USA, vol. 101, pp. 12814-12817, 2004.

[13] X. Xiong, Y. Hanein, J. Fang, Y. Wang, W. Wang, D. T. Schwartz, and K. F. Bohringer, "Controlled multibatch self-assembly of microdevices," J. Microelectromech. Syst., vol. 12, pp. 117-127, 2003.

[14] J. Chung, W. Zheng, and H. O. Jacobs, "Programmable reconfigurable self-assembly: approaching the parallel heterogeneous integration on flexible substrates," presented at the IEEE International Conference on Micro Electro Mechanical Systems (MEMS), Miami Beach, FL, 2005, unpublished.

[15] W. Zheng, J. Chung, and H. O. Jacobs, "Non-robotic fabrication of packaged microsystems by shape-and-solder-directed self-assembly," presented at the IEEE International Conference on MEMS, Miami Beach, FL, Jan. 30, 2005, unpublished.

[16] M. Walz, "Component rework: a small world and getting smaller," Circuits Assembly, vol. 1, pp. 32-37, 2003.

[17] K. S. Birdi, Self-Assembly Monolayer Structures of Lipids and Macromolecules at Interfaces. New York: Kluwer Academic/Plenum, 1999.

[18] W. Zheng and H. O. Jacobs, "Shape-and-solder-directed self-assembly to package semiconductor device segments," Appl. Phys. Lett., vol. 85, pp. 3635-3637, 2004.

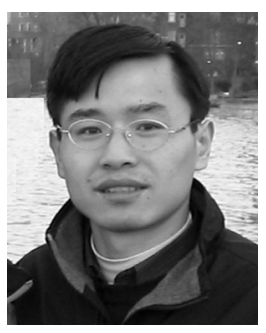

Wei Zheng (M'05) received the M.S. degree in precision instruments from Tsinghua University, China, in 1999. He is currently pursuing the Ph.D. degree in electrical engineering at the University of Minnesota-Twin Cities.

His research interests include fluidic self-assembly, microelectronics packaging, MEMS, and nanotechnology.

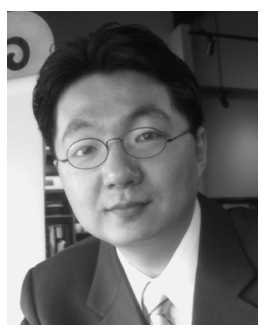

Jaehoon Chung (S'97-M'06) received the B.S. and M.S. degrees in electrical engineering from Korea University in 1996 and 1998, respectively. After receiving M.S., he had been a MEMS process researcher in LG Electronics Institute of Technology. $\mathrm{He}$ has been a graduate student pursuing the Ph.D. degree in electrical engineering at the University of Minnesota-Twin Cities since 2003.

His research interests include fluidic selfassembly, microfabrication on planar and nonplanar surface, silicon optical bench, microelectronics packaging, and other topics in MEMS and NEMS device design and fabrication.

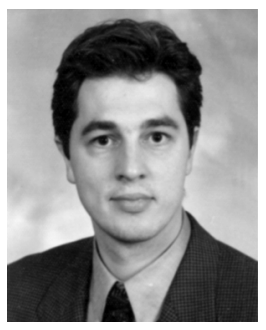

Heiko O. Jacobs (M'02) received the M.Sc. degree in electrical engineering from the University of Wuppertal, Germany, in 1995 and the Doctoral degree (Dr.sc.Techn.) in engineering from the Swiss Federal Institute of Technology (ETH), Zurich, Switzerland, in 1999.

$\mathrm{He}$ joined the faculty at the University of Minnesota-Twin Cities in 2001 as an Assistant Professor following a postdoctoral research position at Harvard University, Cambridge, MA. During his academic career, he has carried out research at different departments-Chemistry (Harvard), Mechanical Engineering (ETH), Physics (ETH), and Electrical Engineering (ETH) - in interdisciplinary groups, all working in areas of micro- and nanotechnology. His research at the University of Minnesota focuses on nontraditional micro and nanotechnologies-integration of nanomaterials and devices across length scales and material boundaries. The current research projects are in the areas of self-assembly based manufacturing, chip scale heterogeneous integration, nanotechnology, nanoparticle-based devices, directed growth of nanowires, and nanometer-scale charge-based printing (nanoxerography). 